\title{
Time-dependent evolution of two coupled Luttinger liquids
}

\author{
E. Perfetto \\ Instituto de Estructura de la Materia, Consejo Superior de Investigaciones Científicas, Serrano 123, 28006 Madrid, Spain \\ and Istituto Nazionale di Fisica Nucleare, Laboratori Nazionali di Frascati, Via E. Fermi 40, 00044 Frascati, Italy
}

(Received 27 July 2006; revised manuscript received 13 October 2006; published 30 November 2006)

\begin{abstract}
We consider two disconnected Luttinger liquids which are coupled at $t=0$ through chiral density-density interactions. Both for $t<0$ and $t \geqslant 0$ the system is exactly solvable by means of bosonization and this allows us to evaluate analytically the time dependence of correlation functions. We find that in the long-time limit the critical exponent governing the one-particle correlation function differs from the exponent dictated by the equilibrium ground state of the coupled system. We also discuss how this reflects on some physical quantities which are accessible to real experiments.
\end{abstract}

DOI: 10.1103/PhysRevB.74.205123

PACS number(s): 71.10.Hf, 73.63.Fg, 73.22.-f

Time-dependent quantum systems have attracted much attention recently. This is because the dynamical response of nanoscopic devices is now accessible in many experiments with relevance in practical applications such as quantum computing and single-electron transport. ${ }^{1}$ On one side we discuss the wide investigation of out-of-equilibrium phenomena in transport experiments, where time-dependent transient currents are measured at picosecond time scales. ${ }^{2}$ On the other side, recent experiments in ultracold atoms confined in optical lattices have shown that it is possible to time tune the strength of the interactions in both bosonic ${ }^{3}$ and fermionic ${ }^{4}$ systems, by using the so-called Feshbach resonance. In these cases, the intriguing question arises: what is the steady state to which the initial ground state relaxes to $?^{5,6}$

In light of this challenging physics, the theoretical investigation of the time-dependent evolution of many-body interacting system deserves special attention. Recently, an exact formulation of time-dependent transport in electronic systems ${ }^{7}$ was derived in the framework of time-dependentdensity-functional theory. ${ }^{8}$ Unfortunately, its implementation to strongly interacting systems is hard. An efficient numerical tool to address the problem of the electron-electron $(e$ $-e)$ interactions is the time-dependent density-matrixrenormalization-group method, ${ }^{9-11}$ which turns out to have excellent performance in one-dimensional (1D) systems. For what concerns electronic systems with time-dependent parameters but not involving charge transport, in a recent paper Cazalilla calculated the exact evolution of a noninteracting 1D system following a sudden switch on of Luttinger liquid $e-e$ interaction. ${ }^{12}$ Remarkably, it is found that the initial ground state reaches a stationary state only if the system is infinite sized and its asymptotic behavior differs from that of the interacting equilibrium system. ${ }^{6}$

In this paper, we extend this analysis to two coupled interacting 1D systems. Our system consists of two isolated Luttinger liquids which are connected at $t=0$ through interactions between chiral electron densities located at different liquids.

The Luttinger liquid is the prototype of interacting electrons in 1D and is governed by the so-called TomonagaLuttinger Hamiltonian. In this model, the electrons have a linear dispersion relation around positive (right) and negative (left) Fermi points located at $\pm k_{F}$, and the $e$ - $e$ interactions act only between right/left electron densities. This means that only the component with momentum transfer $p \sim 0$ of the Coulomb repulsion is retained, while the $p \sim 2 k_{F}$ component is assumed to be negligible. The model is exactly solvable by means of the bosonization technique, which allows us to write the electron Hamiltonian in terms of boson operators b's.

In the following we shall adopt a similar notation as in Ref. 12 .

For $t<0$, the system consists of two disconnected identical interacting electron liquids described by the bosonized Hamiltonian $^{13} H_{0}=H_{1}+H_{2}$ with

$$
\begin{aligned}
H_{i}= & \frac{1}{2} \sum_{q \neq 0}\left[\left(v_{F}+g_{4}^{(4)}(q)\right)|q|\left(b_{i}^{\dagger}(q) b_{i}(q)+b_{i}(q) b_{i}^{\dagger}(q)\right)\right. \\
& \left.-g_{4}^{(2)}(q)\left(b_{i}^{\dagger}(q) b_{i}^{\dagger}(-q)+b_{i}(q) b_{i}(-q)\right)\right],
\end{aligned}
$$

where $i=1,2,\left[b_{i}(q), b_{j}^{\dagger}\left(q^{\prime}\right)\right]=\delta_{i, j} \delta_{q, q^{\prime}}, v_{F}$ is the Fermi velocity, $g_{4}^{(4)}(q)$ is the interaction parameter between right-right (positive $q$ ) and left-left (negative $q$ ) electron densities, while $g_{4}^{(2)}(q)$ is the interaction parameter between left-right densities.

The system is diagonalized by the well-known Bogoliubov transformation:

$$
\begin{aligned}
& \tilde{b}_{i}(q)=\cosh \varphi(q) b_{i}(q)+\sinh \varphi(q) b_{i}^{\dagger}(-q), \\
& \tilde{b}_{i}^{\dagger}(q)=\sinh \varphi(q) b_{i}(-q)+\cosh \varphi(q) b_{i}^{\dagger}(q),
\end{aligned}
$$

with

$$
\tanh 2 \varphi(q)=g_{4}^{(2)}(q) /\left[v_{F}+g_{4}^{(4)}(q)\right],
$$

and renormalized velocity

$$
v(q)=\sqrt{\left(v_{F}+g_{4}^{(4)}(q)\right)^{2}-\left(g_{4}^{(2)}(q)\right)^{2}} .
$$

For $t \geqslant 0$, the chiral density-density interactions between the two Luttinger liquids are switched on and the system is governed by the total Hamiltonian

$$
H_{\mathrm{tot}}=H_{0}+\theta(t) H_{12},
$$

where 


$$
\begin{aligned}
H_{12}= & \sum_{q \neq 0}\left[g _ { 2 } ^ { ( 4 ) } ( q ) \left(b_{1}^{\dagger}(q) b_{2}(q)+b_{2}^{\dagger}(q) b_{1}(q)+b_{1}(q) b_{2}^{\dagger}(q)\right.\right. \\
& +b_{2}(q) b_{1}^{\dagger}(q)-g_{2}^{(2)}(q)\left(b_{1}^{\dagger}(q) b_{2}^{\dagger}(-q)+b_{1}(q) b_{2}(-q)\right. \\
& \left.\left.+b_{2}^{\dagger}(q) b_{1}^{\dagger}(-q)+b_{2}(q) b_{1}(-q)\right)\right],
\end{aligned}
$$

where $g_{2}^{(4)}(q)\left(g_{2}^{(2)}(q)\right)$ is the interaction parameter between electron densities of the same (opposite) chirality in different liquids. Physically, $H_{12}$ could represent the long-range component of the Coulomb repulsion felt by electrons located in two (quasi) 1D metallic systems close to each other. Here we are considering a general situation where $g_{2}^{(4)}(q) \neq g_{2}^{(2)}(q)$ but in real systems like carbon nanotubes, they coincide (see below). It is worthwhile to remark that in the present model the coupling between the two liquids does not involve any interliquid electron tunneling.

$H_{\text {tot }}$ is again diagonalized by introducing symmetrized and antisymmetrized operators: ${ }^{14}$

$$
\begin{aligned}
& s(q)=\left[b_{1}(q)+b_{2}(q)\right] / \sqrt{2}, \\
& a(q)=\left[b_{1}(q)-b_{2}(q)\right] / \sqrt{2} .
\end{aligned}
$$

In terms of Eqs. (7) $H_{\text {tot }}$ decouples in two independent nonequivalent Luttinger liquids:

$$
\begin{aligned}
H_{\mathrm{tot}}= & H_{s}+H_{a} \\
= & \frac{1}{2} \sum_{q \neq 0}\left[\left(v_{F}+g_{4}^{(4)}(q)+g_{2}^{(4)}\right)|q|\left(s^{\dagger}(q) s(q)+s(q) s^{\dagger}(q)\right)\right. \\
& \left.-\left(g_{4}^{(2)}(q)+g_{2}^{(2)}(q)\right)\left(s^{\dagger}(q) s^{\dagger}(-q)+s(q) s(-q)\right)\right] \\
& +\frac{1}{2} \sum_{q \neq 0}\left[\left(v_{F}+g_{4}^{(4)}(q)-g_{2}^{(4)}\right)|q|\left(a^{\dagger}(q) a(q)+a(q) a^{\dagger}(q)\right)\right. \\
& \left.-\left(g_{4}^{(2)}(q)-g_{2}^{(2)}(q)\right)\left(a^{\dagger}(q) a^{\dagger}(-q)+a(q) a(-q)\right)\right] .
\end{aligned}
$$

Finally, the diagonal form of $H_{\text {tot }}$ is obtained in terms of

$$
\begin{aligned}
& \tilde{s}(q)=\cosh \varphi_{s}(q) s(q)+\sinh \varphi_{s}(q) s^{\dagger}(-q), \\
& \tilde{s}^{\dagger}(q)=\sinh \varphi_{s}(q) s(-q)+\cosh \varphi_{s}(q) s^{\dagger}(q), \\
& \tilde{a}(q)=\cosh \varphi_{a}(q) a(q)+\sinh \varphi_{a}(q) a^{\dagger}(-q), \\
& \tilde{a}^{\dagger}(q)=\sinh \varphi_{a}(q) a(-q)+\cosh \varphi_{a}(q) a^{\dagger}(q),
\end{aligned}
$$

where

$$
\tanh 2 \varphi_{s, a}(q)=\left[g_{4}^{(2)}(q) \pm g_{2}^{(2)}(q)\right] /\left[v_{F}+g_{4}^{(4)}(q) \pm g_{2}^{(4)}(q)\right],
$$

and renormalized velocities

$$
v_{s, a}(q)=\sqrt{\left(v_{F}+g_{4}^{(4)}(q) \pm g_{2}^{(4)}(q)\right)^{2}-\left(g_{2}^{(2)}(q) \pm g_{4}^{(2)}(q)\right)^{2}} .
$$

Now we are in the position to evaluate the equal-time one-particle correlation function for $t>0$, defined as

$$
G_{\gamma}^{(i)}(x, t)=\left\langle e^{i H_{\mathrm{tot}^{t}}} \psi_{i, \gamma}(x) \psi_{i, \gamma}^{\dagger}(0) e^{-i H_{\mathrm{tot}}{ }^{t}}\right\rangle_{H_{0}},
$$

where $i=1,2$ labels the two Luttinger liquids decoupled at $t<0$, the subscript $\gamma=R, L$ indicates the right/left character of the noninteracting fermion fields $\psi$ and $\psi^{\dagger}$, and $\langle\cdots\rangle_{H_{0}}$ is the zero-temperature average in the ensemble $H_{0}$. Without any loss of generality we shall focus on $G_{R}^{(1)}(x, t)$.

The bosonization technique allows us to calculate $G_{R}$ with logarithmic accuracy, which is exact for distances/times much longer than the typical range of the interactions. The key point of bosonization is that it is possible to express the fermion field in terms of boson fields. For instance, the rightmover fermion field is given by the following expression:

$$
\psi_{i, R}(x)=\frac{\eta_{R}}{(2 \pi \alpha)^{1 / 2}} e^{i \Phi_{i, R}(x)},
$$

where $\alpha$ is a short-distance cutoff, proportional to the lattice spacing, $\eta_{R}$ is an anticommuting Klein factor, and

$$
\begin{aligned}
\Phi_{i, R}(x)= & \sum_{q>0}\left(\frac{2 \pi}{q L}\right)^{1 / 2} e^{-\alpha q / 2}\left[b_{i}^{\dagger}(q) e^{-i q x}+b_{i}(q) e^{i q x}\right] \\
& +\varphi_{0, R}+2 \pi x N_{R} / L,
\end{aligned}
$$

where $N_{R}$ is the total number of right electrons, $\left[\varphi_{0, R}, N_{R}\right]$ $=i$, and $L$ is the length of the system.

Thus the computation of the correlation function reduces to

$$
\begin{aligned}
G_{R}^{(1)}(x, t) & =\frac{1}{2 \pi \alpha}\left\langle e^{i \Phi_{1, R}(x, t)} e^{-i \Phi_{1, R}(0, t)}\right\rangle_{H_{0}} \\
& =\frac{1}{2 \pi \alpha} e^{-1 / 2\left\{\left\langle\left(\Phi_{1, R}(x, t)-\Phi_{1, R}(0, t)\right)^{2}\right\rangle_{H_{0}}-\left[\Phi_{1, R}(x, t), \Phi_{1, R}(0, t)\right]\right\}},
\end{aligned}
$$

where $\Phi_{1, R}(x, t)=e^{i H_{\text {tot }} t} \Phi_{1, R}(x) e^{-i H_{\text {tot }} t}$. In order to compute $\Phi_{1, R}(x, t)$, it is convenient to first evaluate $b_{1}(q, t)$ $=e^{i H_{\mathrm{tot}}{ }^{t}} b_{1}(q) e^{-i H_{\mathrm{tot}}{ }^{t}}$ in terms of the $\tilde{b}_{i}(q)$ 's and $\tilde{b}_{i}^{\dagger}(q)$ 's, since they diagonalize $H_{0}$ which defines the ensemble we average on.

After a lengthy algebra involving a direct and an inverse Bogoliubov transformation in Eq. (9), and the inverse of Eq. (2), one finds

$$
\begin{aligned}
b_{1}(q, t)= & A(q, t) \tilde{b}_{1}(q)+B^{\star}(q, t) \tilde{b}_{1}^{\dagger}(-q)+C(q, t) \tilde{b}_{2}(q) \\
& +D^{\star}(q, t) \tilde{b}_{2}^{\dagger}(-q),
\end{aligned}
$$

where

$$
\begin{aligned}
A(q, t)= & \cosh \varphi(q)\left[-i \sin v_{s}|q| t \cosh 2 \varphi_{s}(q)+\cos v_{s}|q| t\right. \\
& +(a \leftrightarrow s)]-\sinh \varphi(q)\left[-i \sin v_{s}|q| t \sinh 2 \varphi_{s}(q)\right. \\
& +(a \leftrightarrow s)], \\
B^{\star}(q, t)= & -\sinh \varphi(q)\left[-i \sin v_{s}|q| t \cosh 2 \varphi_{s}(q)+\cos v_{s}|q| t\right. \\
& +(a \leftrightarrow s)]+\cosh \varphi(q)\left[-i \sin v_{s}|q| t \sinh 2 \varphi_{s}(q)\right. \\
& +(a \leftrightarrow s)],
\end{aligned}
$$




$$
\begin{aligned}
C(q, t)= & \cosh \varphi(q)\left[-i \sin v_{s}|q| t \cosh 2 \varphi_{s}(q)+\cos v_{s}|q| t\right. \\
& -(a \leftrightarrow s)]-\sinh \varphi(q)\left[-i \sin v_{s}|q| t \sinh 2 \varphi_{s}(q)\right. \\
& -(a \leftrightarrow s)], \\
D^{\star}(q, t)= & -\sinh \varphi(q)\left[-i \sin v_{s}|q| t \cosh 2 \varphi_{s}(q)+\cos v_{s}|q| t\right. \\
& -(a \leftrightarrow s)]+\cosh \varphi(q)\left[-i \sin v_{s}|q| t \sinh 2 \varphi_{s}(q)\right. \\
& -(a \leftrightarrow s)] .
\end{aligned}
$$

The above relations allow us to evaluate exactly the time dependence in the correlation function, which reads

$$
\begin{aligned}
G_{R}^{(1)}(x, t)= & \frac{1}{2 \pi \alpha} \exp \left[-\sum_{q>0}\left(\frac{2 \pi}{q L}\right) e^{-\alpha q} \times\left[\left(|A(q, t)|^{2}\right.\right.\right. \\
& \left.+|C(q, t)|^{2}\right)(-i \sin q x+1-\cos q x)+\left(|B(q, t)|^{2}\right. \\
& \left.\left.\left.+|D(q, t)|^{2}\right)(i \sin q x+1-\cos q x)\right]\right]
\end{aligned}
$$

The sum over $q$ is performed by using $q=2 \pi n / L$ and we end up with

$$
\begin{aligned}
G_{R}^{(1)}(x, t)= & \frac{c}{d(x)^{1 / 4\left[2 \cosh 2 \varphi+\cosh \left(2 \varphi-4 \varphi_{s}\right)+\cosh \left(2 \varphi-4 \varphi_{a}\right)\right]}} \\
& \times\left[\frac{d\left(x-2 v_{s} t\right) d\left(x+2 v_{s} t\right)}{d\left(2 v_{s} t\right)^{2}}\right]^{1 / 8\left[\cosh \left(2 \varphi-4 \varphi_{s}\right)-\cosh 2 \varphi\right]} \\
& \times\left[\frac{d\left(x-2 v_{a} t\right) d\left(x+2 v_{a} t\right)}{d\left(2 v_{a} t\right)^{2}}\right]^{1 / 8\left[\cosh \left(2 \varphi-4 \varphi_{a}\right)-\cosh 2 \varphi\right]},
\end{aligned}
$$

where $d(x)=L \sin (\pi x / L)$. In order to obtain the above expression, we have introduced momentum-independent $\varphi$ and $\varphi_{s, a}$, such that $\sinh f(q) \approx e^{-R_{0}|q| / 2} \sinh f(0)$, with $R_{0}$ of the order of the range of the $e$-e interactions; in this way the finite overall prefactor $c$ is produced. ${ }^{15}$

Let us now consider some relevant limits. First, it is straightforward to check that in the noninteracting case (i.e. $\varphi=\varphi_{s}=\varphi_{a}=0$ and $\left.v_{a}=v_{s}=v_{F}\right)$ we recover

$$
G_{R}^{(1)}(x, t)=\frac{c}{d(x)}
$$

on the other hand, for $t \rightarrow 0$ we find

$$
G_{R}^{(1)}(x, 0)=\frac{c^{\prime}}{d(x)^{\cosh 2 \varphi}},
$$

where $c^{\prime}$ is again a finite constant. This result corresponds to the correlation function of an individual Luttinger liquid governed by $H_{1}$ or $H_{2}$, as it should.

More interesting is the case in which $g_{4}^{(2)}=g_{4}^{(4)}=g_{2}^{(4)}=0$ (i.e. $\varphi=0$ and $-\varphi_{a}=\varphi_{s} \equiv \bar{\varphi} \neq 0$ and $v_{a}=v_{s} \equiv \bar{v} \neq v_{F}$ ). In this case, the system corresponds to two identical replicas of the model considered by Cazalilla, i.e. an isolated noninteracting $1 \mathrm{D}$ system with density-density interactions switched on at $t=0$. In this case we obtain

$$
\begin{aligned}
G_{R}^{(1)}(x, t)= & \frac{c}{d(x)^{1 / 4(\cosh 4 \bar{\varphi}+1)}} \\
& \times\left[\frac{d(x-2 \bar{v} t) d(x+2 \bar{v} t)}{d(2 \bar{v} t)^{2}}\right]^{1 / 4(\cosh 4 \bar{\varphi}-1)},
\end{aligned}
$$

which recovers the result of Cazalilla [Eq. (9) of Ref. 12].

The most remarkable result is obtained from the long-time limit $t \rightarrow \infty$ of Eq. (19). In this case we find

$$
G_{R}^{(1)}(x) \approx \frac{c}{|x|^{1 / 4\left[2 \cosh 2 \varphi+\cosh \left(2 \varphi-4 \varphi_{s}\right)+\cosh \left(2 \varphi-4 \varphi_{a}\right)\right]}},
$$

where we have also taken $L \rightarrow \infty$. We point out that if the infinite length limit is not taken, the correlations obey an oscillatory behavior even at long time, confirming that in a finite-sized system a steady state is not reachable. However, as already discussed in Ref. 12, in mesoscopic systems with finite $L$ (e.g., $L \sim 1 \mu \mathrm{m}$ for carbon nanotubes) a genuine stationary state can be reached because of finite temperature effects. Indeed, if the temperature $T$ is larger than the level spacing $\Delta \varepsilon \sim v_{F} / L$, we have to replace $L$ by $v_{F} / T$ and $\sin$ by sinh in the definition of $d(x)$. As a consequence, for very large distances/times, the Green function decays exponentially as $G_{R}^{1} \sim e^{-|x| \nu T}$, where $\nu$ is the same exponent displayed in the power law at $T=0 .{ }^{16}$ Thus it appears that in the exponential regime we can still keep track of the Luttinger liquid correlations encoded by $\nu$. On the other hand, for distances/ times smaller than the thermal length $v_{F}^{\prime} / T$ (with $v_{F}^{\prime}$ the renormalized Fermi velocity ${ }^{16}$ ) the power law discussed above still holds.

In the thermodynamic limit, it is worthwhile to notice that the result in Eq. (23) does not coincide with the equilibrium equal-time correlation function of two coupled Luttinger liquids governed by $H_{0}+H_{12}$. Indeed, in the latter case, the correlation function reads

$$
G_{R}^{(1)}(x) \approx \frac{c^{\prime \prime}}{|x|^{1 / 2\left[\cosh \left(2 \varphi_{s}\right)+\cosh \left(2 \varphi_{a}\right)\right]}},
$$

with finite $c^{\prime \prime}$. The difference in the critical exponents in the two cases reveals that in the long-time limit the ground state of $H_{0}$ evolved by $H_{0}+\theta(t) H_{12}$ reaches a stationary state which is not the ground state of $H_{0}+H_{12}$. This is due to the fact that for $t>0$, the energy is conserved and therefore the system cannot relax to the ground state. Indeed the critical exponent in Eq. (23) is larger than the critical exponent in Eq. (24), consistent with the fact that in the latter case the system is able to optimize the repulsive interactions given by $H_{12}$.

Now let us discuss the physical consequences of this result. To this end, we introduce the critical exponent $\alpha$ governing some relevant observables such as the tunneling density of states $\rho,{ }^{17}$

$$
\rho(\omega) \sim \omega^{\alpha},
$$

which is detectable with scanning tunneling microscopy by probing the bulk 1D conductor. ${ }^{18,19}$

In order to compare to real systems, we have to introduce spin in the previous analysis. This is completely straightfor- 
ward as long as we consider spin-independent $e$-e interactions. For spinless electrons, the $\alpha$ exponent is obtained by subtracting 1 from the exponential one-particle correlation function, while for the corresponding spinful system we just have to halve such a value. ${ }^{25}$

It is worthwhile to note that, while in equilibrium systems, the critical exponent of $\rho(\omega)$ can be safely extracted from the equal-time one-particle Green's function; in out-ofequilibrium situations such a procedure is far from obvious. ${ }^{20}$ Therefore, it would be convenient to relate $\alpha$ to some other energy-independent observable, such as the momentum distribution, which also displays a power law: ${ }^{17}$

$$
n(q) \propto\left|q-k_{F}\right|^{\alpha} .
$$

$n(q)$ is accessible, for instance, from angle-resolved photoemission spectroscpy, which has been successfully applied to quasi-1D materials ${ }^{21,22}$ to measure $\alpha^{23,24}$ We also mention that such a technique is not affected by the uncertainties regarding the contacts, which are present in transport experiments. At this point, we note again that the above power law would be cut off by finite temperature effects if $\left|q-k_{F}\right|$ $\sim T / v_{F}$.

From Eqs. (23) and (24), the exponent governing $n(q)$ in the two different cases discussed above read

$$
\begin{aligned}
\alpha_{\text {asympt }}= & \frac{1}{8}\left[2 \cosh 2 \varphi+\cosh \left(2 \varphi-4 \varphi_{s}\right)+\cosh \left(2 \varphi-4 \varphi_{a}\right)\right] \\
& -\frac{1}{2} \\
& \alpha_{\text {equil }}=\frac{1}{4}\left[\cosh \left(2 \varphi_{s}\right)+\cosh \left(2 \varphi_{a}\right)\right]-\frac{1}{2} .
\end{aligned}
$$

The model we have considered could find an experimental realization in 1D fermionic systems built within optical potential. Unfortunately, in these systems the interaction is short ranged and this makes the term $H_{12}$ hard to realize. Long-range dipole-dipole interactions have been recently obtained in chromium bosonic atoms ${ }^{26}$ and Fermi gases with long-range interactions are likely becoming available.

On the other hand, it is tempting to relate our results to the physics of carbon nanotubes. Metallic carbon nanotubes are believed to be rather good (although approximate) realizations of Luttinger liquids, since in normal conditions the main correlation effects come from the long-range part of the Coulomb repulsion (through the $g_{i}^{(j)}$ parameters), while the back-scattering interactions with large momentum transfer suffer a $1 / R$ suppression, where $R$ is the radius of the nanotube. ${ }^{18,19}$ Indeed, back-scattering interactions are marginal operators in the renormalization group sense, and in carbon nanotubes are predicted to break down the Luttinger liquid state only at very low energy scales. ${ }^{18}$ This is supported by direct observation of power-law decay of the linear conductance as a function of temperature. ${ }^{27-29}$

It is worthwhile to recall that each individual carbon nanotube is itself composed by two coupled (identical) Luttinger liquids, since there is a left and a right branch, respectively, around each of the two Fermi points. ${ }^{14,18,30}$ This im-

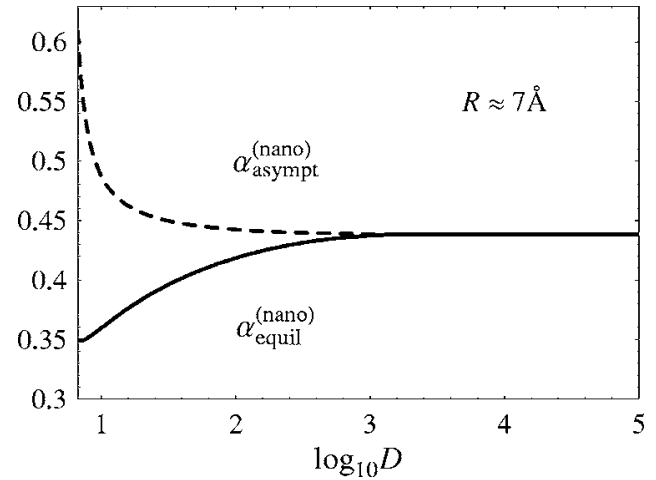

FIG. 1. Plot of the critical exponents $\alpha_{\text {asympt }}^{\text {(nano) }}$ (dashed curve) and $\alpha_{\text {equil }}^{\text {(nano) }}$ (solid curve) for typical $(10,10)$ nanotubes as a function of the logarithm of the distance $D$ between the nanotubes. In $\log _{10} D$, $D$ is expressed in $\AA$.

plies that the critical exponent governing the decay of the correlation functions in these systems is actually one half the exponents defined in Eqs. (27). Therefore, in the following we will use $\alpha_{\text {asympt }}^{\text {(nano) }}=\alpha_{\text {asympt }} / 2$ and $\alpha_{\text {equil }}^{\text {(nano) }}=\alpha_{\text {equil }} / 2$.

We will compare two possible measurements realized in two different experimental setups. The first system (setup A), which gives access to $\alpha_{\text {asympt }}^{\text {(nano) }}$, is made by two (initially distant) nanotubes which can be quickly aligned at a controlled distance. This makes it possible to mimic the switching on of intertube interactions. In this case, of course, the critical exponent could only be measured in the final steady state, and any transient regime is definitively unaccessible. The second system (setup B), which gives access to $\alpha_{\text {equil }}^{\text {(nano) }}$, consists in having the carbon nanotubes synthesized at a given distance, for instance in a double-wall nanotube. In this case, the system has fixed intertube interactions built in from the very beginning.

Typical metallic nanotubes correspond to armchair (10, 10) geometry (i.e. radius $R \approx 7 \AA$ ) with length $L \sim 1 \mu \mathrm{m}$. The strength of the density-density Coulomb interactions is then ${ }^{18,30}$

$$
\begin{aligned}
& g_{4}^{(2)}(q \sim 0)=g_{4}^{(4)}(q \sim 0) \approx \frac{4 e^{2}}{\kappa \pi v_{F}} K_{0}\left(q_{c} R\right), \\
& g_{2}^{(2)}(q \sim 0)=g_{2}^{(4)}(q \sim 0) \approx \frac{4 e^{2}}{\kappa \pi v_{F}} K_{0}\left(q_{c} D\right),
\end{aligned}
$$

where $K_{0}$ is the modified Bessel function, $q_{c}=2 \pi / L$ is the infrared momentum cutoff due to the finite size of the nanotubes, $D \geq R$ is the intertube distance, $\kappa \sim 2$ is the dielectric constant of typical nanotubes, and $v_{F} \approx 8 \times 10^{5} \mathrm{~m} / \mathrm{s}$. We recall at this point that the amplitude of intratube backscattering interactions is approximatively ${ }^{18} 0.1 e^{2} a / \kappa \pi v_{F} R$ (with $a=2.46 \AA$ ), which is much smaller than both intra- and intertube forward scattering interactions for $D \geq R$. We also mention that single-electron tunneling between nanotubes is usually strongly suppressed since the misalignment of the respective carbon lattices prevents momentum conservation 
in the tunneling process. Therfore, the experimental setups that we propose could be fairly approximated by the model of two coupled Luttinger liquids.

By inserting $g_{i}^{(j)}$ obtained in Eqs. (28) into Eqs. (3) and (10), we can estimate the critical exponents accessible to experiments within setups A and B.

The two exponents are plotted in Fig. 1 as a function of the distance between the nanotubes. For $D \rightarrow \infty$, the intertube couplings $g_{2}^{(4)}$ and $g_{2}^{(2)}$ vanish [see Eq. (28)] and $\alpha_{\text {asympt }}^{(\text {nano) }}$ and $\alpha_{\text {equil }}^{\text {(nano) }}$ tend to the common value

$$
\alpha_{\text {disc }}^{(\text {nano })}=\frac{1}{4}(\cosh 2 \varphi-1) \approx 0.43,
$$

which governs the correlations of two completely independent nanotubes, according to Eq. (21). This value is in agreement with previous estimates given for single typical nanotubes. ${ }^{18,19}$

When the two nanotubes are brought close to each other in setup A (Fig. 1, dashed line), the intertube interactions are switched on and $\alpha_{\text {asympt }}^{(\text {nano) }}$ increases. In particular, at $D \sim R$ we find

$$
\alpha_{\text {asympt }}^{\text {(nano) }} \approx 0.60 \text {. }
$$

Regarding setup B (Fig. 1, solid line), if the two carbon nanotubes have been synthesized at the same distance $D$ $\sim R$, the predicted exponent is

$$
\alpha_{\text {equil }}^{(\text {nano })} \approx 0.35,
$$

which is significantly smaller than $\alpha_{\text {asympt }}^{\text {(nano) }}$. We believe that such a large difference could be detected, even though the appropriate experimental setup might be difficult to realize. In particular, in setup A, the two nanotubes should approach each other at a speed comparable to (or even higher than) the Fermi velocity $\left(\sim 10^{6} \mathrm{~m} / \mathrm{s}\right)$. We note, by the way, that this problem does not arise in cold atomic systems, where the Fermi velocity is orders of magnitude smaller.

In conclusion, we have computed the time-dependent evolution of the single-particle correlation function of two Luttinger liquids coupled by a sudden switch on of the interliquid interaction. This allows us to evaluate the critical exponent $\alpha$ governing some physical observables accessible to real experiments, such as the momentum distribution function. We find that in the long-time limit, the initial ground state relaxes to a stationary state which is not the equilibrium ground state of the coupled system. In the latter case, the critical exponent $\alpha_{\text {equil }}$ that results is smaller than the asymptotic exponent $\alpha_{\text {asympt }}$ by about a factor of 2 . An experiment capable of detecting such a remarkable finding in carbon nanotubes is proposed. Finally, we believe that the present study could be also relevant in experiments involving ultracold fermionic atoms loaded in optical lattices, where tunable Luttinger liquids might be realized in the near future.

The author kindly acknowledges helpful discussions with J. González, M. Cini, and G. Stefanucci. This work has been supported by Ministerio de Educación y Ciencia (Spain) through Grant Nos. FIS2005-05478-C02-01/02 and by INFN (Italy) through Grant No. 10068.
${ }^{1}$ T. Fujisawa, T. Hayashi, and S. Sasaki, Rep. Prog. Phys. 69, 759 (2006).

${ }^{2}$ T. Fujisawa, Y. Tokura, and Y. Hirayama, Phys. Rev. B 63, 081304(R) (2001).

${ }^{3}$ M. Greiner et al., Nature (London) 415, 39 (2002); T. Stöferle et al., Phys. Rev. Lett. 92, 130403 (2004).

${ }^{4}$ H. Ott et al., Phys. Rev. Lett. 92, 160601 (2004); M. Köhl et al., ibid. 94, 080403 (2005).

${ }^{5}$ T. Kinoshita, T. Wenger, and D. S. Weiss, Nature (London) 440, 900 (2006)

${ }^{6}$ M. Rigol, V. Dunjko, V. Yurovskii, and M. Olshanii, cond-mat/ 0604476 (unpublished).

${ }^{7}$ M. Cini, Phys. Rev. B 22, 5887 (1980).

${ }^{8}$ G. Stefanucci and C. O. Almbladh, Phys. Rev. B 69, 195318 (2004).

${ }^{9}$ M. A. Cazalilla and J. B. Morton, Phys. Rev. Lett. 88, 256403 (2002).

${ }^{10}$ S. R. White and A. E. Feiguin, Phys. Rev. Lett. 93, 076401 (2004).

${ }^{11}$ A. J. Daley, C. Kollath, U. Schollwöck, and G. Vidal, J. Stat. Mech.: Theory Exp. (2004) P04005.

${ }^{12}$ M. A. Cazalilla, Phys. Rev. Lett. 97, 156403 (2006).

${ }^{13}$ J. Gonzàlez et al., Quantum Electron Liquids and High-T $T_{c}$ Superconductivity (Springer-Verlag, Berlin, 1995), Chap. 4.

${ }^{14}$ A. Komnik and R. Egger, Phys. Rev. Lett. 80, 2881 (1998).

${ }^{15}$ A. Luther and I. Peschel, Phys. Rev. B 9, 2911 (1974).

${ }^{16}$ T. Giamarchi, Quantum Physics in One Dimension (Oxford Sci- ence Publications, Oxford, 2004), Chap. 3.

${ }^{17}$ J. Voit, J. Phys.: Condens. Matter 5, 8305 (1993).

${ }^{18}$ R. Egger and A. O. Gogolin, Phys. Rev. Lett. 79, 5082 (1997); Eur. Phys. J. B 3, 281 (1998).

${ }^{19}$ C. Kane, L. Balents, and M. Fisher, Phys. Rev. Lett. 79, 5086 (1997).

${ }^{20}$ A. Komnik and A. O. Gogolin, Phys. Rev. B 66, 125106 (2002).

${ }^{21}$ J. Voit et al., Science 290, 501 (2000).

${ }^{22}$ J. Choi, S. M. Lee, Y. C. Choi, Y. H. Lee, and J. C. Jiang, Chem. Phys. Lett. 349, 185 (2001).

${ }^{23}$ H. Ishii et al., Nature (London) 426, 540 (2003).

${ }^{24} \mathrm{We}$ note that in Ref. 23 the photoemission experiment is not angle-resolved.

${ }^{25}$ J. Voit, Rep. Prog. Phys. 58, 977 (1995).

${ }^{26}$ J. Stuhler, A. Griesmaier, T. Koch, M. Fattori, T. Pfau, S. Giovanazzi, P. Pedri, and L. Santos, Phys. Rev. Lett. 95, 150406 (2005).

${ }^{27}$ B. Gao, A. Komnik, R. Egger, D. C. Glattli, and A. Bachtold, Phys. Rev. Lett. 92, 216804 (2004).

${ }^{28}$ Z. Yao, H. W. Postma, L. Balents, and C. Dekker, Nature (London) 402, 273 (1999).

${ }^{29}$ M. Bockrath, D. H. Cobden, J. Lu, A. G. Rinzler, R. E. Smalley, L. Balents, and P. L. McEuen, Nature (London) 397, 598 (1999).

${ }^{30}$ J. Gonzàlez and E. Perfetto, Phys. Rev. B 72, 205406 (2005); Eur. Phys. J. B 51, 571 (2006). 